\title{
APENDICECTOMIA EM PACIENTES COM IDADE SUPERIOR A 40 ANOS - ANÁLISE DOS RESULTADOS DE 217 CASOS
}

\author{
ANTONIO SÉRGIO BRENNER ${ }^{1}$; JULIANA SANTIN²; FREDERICO VIRMOND NETO²; TANIA BOURSHEID²; \\ RUBENS VALARINI'; RICARDO RYDYGIER ${ }^{1}$ \\ ${ }^{1}$ Hospital Evangélico da Faculdade Evangélica de Medicina do Paraná; \\ ${ }^{2}$ Hospital Universitário Evangélico de Curitiba
}

BRENNER AS; SANTIN J; VIRMOND NETO F; BOURSHEID T; VALARINI R; RYDYGIER R. Apendicectomia em Pacientes com Idade Superior a 40 Anos - Análise dos Resultados de 217 Casos. Rev bras Coloproct, 2006;26(2):128-132.

RESUMO: Introdução: A baixa freqüência de apendicite aguda em pacientes acima de 40 anos indica que uma melhor análise desse grupo torna-se necessária. Método: Realizamos um estudo de Coorte em 1343 pacientes submetidos a apendicectomia entre 2001 a 2005. Duzentos e dezessete pacientes apresentavam idade superior a 40 anos. Os pacientes foram divididos em 2 grupos: pacientes com idade superior $(n=217)$ e inferior $(n=1126)$. O Banco de Dados do Serviço em Cirurgia do Hospital Universitário Evangélico foi utilizado para fornecer os achados operatórios, os exames histopatológicos e o resultado clínico imediato. Resultados: No grupo com idade acima de 40 anos, os achados cirúrgicos demonstraram o apêndice em fase edematosa em $42,85 \%$ e em $26,72 \%$ foi identificada supuração. A incisão mediana foi realizada em 39,17\% e o tempo de internação médio foi de 4,65 dias. No grupo com idade abaixo de 40 anos, os achados cirúrgicos demonstraram o apêndice em fase edematosa em 24,5\% e supuração em 32,6\%. A incisão mediana foi utilizada em $11,90 \%$ dos pacientes e o tempo de internação médio foi de 3,35 dias. Conclusão: Nos pacientes acima de 40 anos, observamos casos mais complexos, provavelmente pelo baixo índice de suspeição.

Descritores: apendicite; apendicectomia; cirurgia; idoso

\section{INTRODUÇÃO}

A apendicite aguda é uma das patologias cirúrgicas mais frequientes. O diagnóstico ainda é notadamente baseado no exame clínico, mas estudos de imagem, como a ultrassonografia, a tomografia computadorizada e exames laboratoriais, como contagem de leucócitos, proteína $\mathrm{C}$ reativa e percentagem de neutrófilos são de grande auxílio diagnóstico. A abordagem cirúrgica ganhou a opção da cirurgia laparoscópica, mas o diagnóstico preciso permanece um desafio. Os sintomas são subjetivos e o exame clínico muito variável. ${ }^{1}$

A apendicite é usualmente uma patologia de jovens. Entretanto, o aumento da sobrevida populacional com desvio da pirâmide etária para uma população cada vez mais idosa, tornou a ocorrência de apendicite aguda em pacientes acima de 40 ou 60 anos mais freqüente. ${ }^{2}$

É importante lembrar que a apendicite aguda apresenta índices elevados de morbidade quando passada despercebida pelo médico menos cauteloso. ${ }^{3}$

Trabalho realizado no Serviço de Coloproctologia do Hospital Universitário Evangélico de Curitiba - HUEC

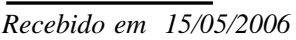

Aceito para publicação em 09/06/2006 
Rev bras Coloproct Abril/Junho, 2006
Apendicectomia em Pacientes com Idade Superior a 40 Anos

- Análise dos Resultados de 217 Casos -

Antonio Sérgio Brenner e Cols.
Vol. 26

$\mathbf{N}^{\circ} 2$
Como agravante, o erro diagnóstico proporciona maior risco nos pacientes portadores de doenças associadas ${ }^{4}$.

Percebemos uma escassez de publicações recentes. Decidimos, portanto, investigar os resultados imediatos do tratamento cirúrgico da apendicite aguda em faixas etárias mais elevadas, nos pacientes do Serviço de Cirurgia Geral e Coloproctologia do Hospital Universitário Evangélico de Curitiba (HUEC).

\section{MATERIAL E MÉTODO}

Foram estudados 1343 pacientes, submetidos a apendicectomia com diagnóstico clínico de apendicite aguda, entre fevereiro de 2001 a outubro de 2005, nos Serviços de Cirurgia Geral e Coloproctologia do Hospital Evangélico de Curitiba. Num estudo retrospectivo de Coorte, 217 apresentavam idade superior ou igual a 40 anos e destes, 37 pacientes apresentavam idade acima de 60 anos.

Para fins comparativos, dividimos os pacientes em 2 grupos: GRUPO > 40 - pacientes acima de 40 anos de idade no momento do diagnóstico e GRUPO < 40 - para os pacientes que no momento diagnóstico apresentavam menos de 40 anos de idade. Também analisamos, isoladamente, os resultados do tratamento cirúrgico de 37 pacientes idosos (acima de 60 anos) de idade.

O Banco de Dados em Coloproctologia do Serviço de Coloproctologia do Hospital Universitário Evangélico de Curitiba (HUEC) foi utilizado para fornecer os seguintes dados: sexo, idade, tempo de internação, tempo de permanência hospitalar, tipo de incisão abdominal, achados operatórios (com ou sem supuração), opção cirúrgica e o resultado cirúrgico imediato. Foram excluídos do estudo os pacientes que foram a óbito durante o seguimento.

Todos os pacientes foram operados pelos médicos do Serviço de Cirurgia Geral e Coloproctologia do HUEC. O diagnóstico foi realizado por avaliação clínica, exames laboratoriais e de imagem pelo cirurgião responsável.

A apendicite foi considerada como edematosa quando o achado operatório foi de edema, hiperemia ou presença de liquido seroso periapendicular, mas ausência de coleção purulenta; ou como supurada, quando o achado foi de coleção purulenta periapendicular, caracterizando apendicite perfurada. Os achados foram determinados pelo cirurgião no momento da laparotomia e confirmados por estudo anatomopatológico.

Não incluímos os casos de apendicectomia laparoscópica na série.

\section{RESULTADOS}

No momento do diagnóstico, 217 pacientes apresentavam idade superior a 40 anos (GRUPO > 40) e 1126, idade inferior (GRUPO < 40). Trinta e sete pacientes apresentavam idade superior a 60 anos. $\mathrm{O}$ tempo médio de internação variou de 1 a 94 dias com média de 2.2 dias. A idade variou de 10 a 84 anos.

No GRUPO > 40, 98 pacientes pertenciam ao sexo feminino e 119, ao masculino. O tempo médio de internação foi de 4.65 dias (1-94). Oitenta e três pacientes $(38,24 \%)$ necessitaram de 4 ou mais dias de internamento. Oitenta e cinco incisões foram medianas $(39,17 \%)$ e $116(53,45 \%)$ tipo McBurney. O achado cirúrgico foi de apendicite edematosa em $93(42,85 \%)$ e supurada em $58(26,72 \%)$. Não foram obtidas informações sobre a fase de inflamação do apêndice em $66(30,41 \%)$ pacientes do GRUPO > 40. (Tabelas $1,2$ e 3$)$

No GRUPO < 40, 554 pacientes eram do sexo masculino e 572 do feminino. O tempo médio de internação foi de 3,35 (1-94) dias. Cento e cinqüenta e um $(13,41 \%)$ pacientes necessitaram de 4 ou mais dias de internação. A incisão utilizada foi do tipo mediana em 134 (11,9\%) e McBurney em 874 (77,61\%). O achado cirúrgico foi de apendicite edematosa em 276 $(24,5 \%)$ e supurada em 368 (32,6\%). Não foram obtidas informações sobre a fase de inflamação do apêndice em $482(42,80 \%)$ pacientes do GRUPO < 40. (Tabelas$1,2$ e 3$)$

Quando comparamos os 1126 pacientes submetidos a apendicectomia, observamos que a freqüência de supuração é menor nos pacientes mais idosos. Por outro lado, a indicação da incisão mediana é maior, bem como o tempo de internação hospitalar.

Trinta e sete pacientes apresentavam mais de 60 anos de idade (variando de 60 a 84 anos - média de 66.1 anos). Em 14 pacientes a incisão foi do tipo McBurney e em 18, do tipo mediana. Não obtivemos informação sobre o tipo de incisão realizada em 5 pacientes. Foi observada supuração em 17 pacientes e 
Tabela 1 - Achados Anatomopatológicos.

\begin{tabular}{lrccc}
\hline Grupo & N & Edematosa & Supurada & Sem Informação \\
\hline GRUPO $>40$ & 217 & $93(42,85 \%)$ & $58(26,72 \%)$ & $66(30,41 \%)$ \\
GRUPO $<40$ & 1126 & $276(24,5 \%)$ & $368(32,6 \%)$ & $482(42,80 \%)$ \\
Acima de 60 anos & 37 & $7(18,9 \%)$ & $17(45,9 \%)$ & $13(35,1)$ \\
\hline
\end{tabular}

Tabela 2 - Tipos de incisões por grupo.

\begin{tabular}{lrrrr}
\hline Grupo & N & Incisão mediana & Incisão McBurney & Sem Informação \\
\hline GRUPO $>40$ & 217 & $85(39,17 \%)$ & $116(53,45 \%)$ & $16(7,37 \%)$ \\
GRUPO < 40 & 1126 & $134(11,90 \%)$ & $874(77,61 \%)$ & $118(10,47 \%)$ \\
Acima de 60 anos & 37 & $18(48,64 \%)$ & $14(37,83 \%)$ & $5(13,51 \%)$ \\
\hline
\end{tabular}

Tabela 3 - Sexo e Idade

\begin{tabular}{llll}
\hline Grupo & N & Masculino & Feminino \\
\hline GRUPO $>40$ & 217 & $119(54,83 \%)$ & $98(45,16 \%)$ \\
GRUPO $<40$ & 1126 & $554(49,20 \%)$ & $572(50,79 \%)$ \\
\hline
\end{tabular}

somente edema apendicular em 7 casos. Nos 18 casos em que a opção cirúrgica foi de laparotomia mediana, observou-se apendicite em fase edematosa (sem abscesso) em apenas 2 pacientes.

\section{DISCUSSÃO}

Apesar da evolução crescente e notável de equipamentos, técnicas cirúrgicas e exames diagnósticos nos últimos 30 anos, na prática poucas mudanças foram observadas no procedimento cirúrgico adotado $^{5}$. A evolução dos exames complementares nos induziria ao raciocínio simplista de que o diagnóstico mais precoce, associado à técnica menos invasiva, resultaria em menores índices de complicações e mortalidade pós-operatória. "Quanto mais as coisas mudam, mais permanecem as mesmas". Com essa frase, HALE et al. em $1997^{3}$ já alertavam a evolução, apenas lenta, do resultado cirúrgico final - fato também observado na cirurgia abdominal em geral.
O diagnóstico diferencial é especialmente importante para os pacientes adultos acima de 40 anos e os mais idosos. As doenças ginecológicas no sexo feminino, a freqüente ocorrência de patologias do sistema urinário, a doença inflamatória intestinal, a síndrome do intestino irritável e até o câncer do cólon confundem o médico atendente no diagnóstico da apendicite aguda, o qual deve lembrar-se desta patologia como uma das possibilidades diagnósticas.

Comparando os resultados atuais com épocas mais longínquas (décadas de 40 e 50), observamos uma marcante melhora em termos de mortalidade. Entretanto, dos anos 60 em diante os resultados parecem não evoluir.

As taxas de laparotomia branca (negativa) tenderiam a diminuir com a evolução dos métodos diagnósticos, mas um fato marcante é a constatação de que postergar a cirurgia não aumenta a mortalidade na apendicite aguda. A cirurgia precoce foi preconizada por muitos e uma taxa de até $20 \%$ para apendicectomia branca era então aceita como "recomendável". Operar precocemente evitaria o risco de perfuração, formação de abscesso e conseqüente peritonite, reduzindo-se a morbidade. Por outro lado, segundo HALE et $\mathrm{al}^{3}$ aguardar até que uma apresentação clínica seja mais convincente, não aumenta a mortalidade. Talvez seja esta a grande mudança na abordagem médica. Permanecemos inseguros no diagnóstico, mas tranquiilos na forma de tratamento e suporte pósoperatório. 
Rev bras Coloproct Abril/Junho, 2006
Apendicectomia em Pacientes com Idade Superior a 40 Anos

- Análise dos Resultados de 217 Casos -

Antonio Sérgio Brenner e Cols.
Vol. 26
O uso da Tomografia Abdominal Computadorizada (TAC) no diagnóstico do abdome agudo (associado ou não à ecografia abdominal) é de utilização crescente na prática ${ }^{6}$. Em relação ao ultrassom, além de coleções intra-abdominais, a TAC possibilita melhor visualização da parede do órgão, diferenciando de possíveis doenças associadas, com provavelmente melhor especificidade e sensibilidade ${ }^{7}$.

Uma nova proposta foi o estabelecimento de um sistema de escores que incorporam o ultrassom, exames clínicos e laboratoriais para auxilio no diagnóstico da apendicite aguda. Quatro variáveis entre 15 analisadas foram correlacionadas à apendicite aguda: Ultrassom que demonstre apendicite aguda; sensibilidade abdominal; contagem de leucócitos e sensibilidade abdominal à descompressão ${ }^{8}$. Yang e colaboradores ${ }^{9}$ chamaram a atenção sobre a importância da dosagem sérica de proteina $\mathrm{C}$ reativa como de auxilio no diagnóstico da apendicite.

Do ponto de vista técnico, a cirurgia vídeoendoscópica foi, provavelmente, a grande evolução. Seu benefício mais evidente é observado em pacientes do sexo feminino, pois possibilita o diagnóstico diferencial com as doenças ginecológicas e na apendicite complicada. A laparoscopia está relacionada a um menor tempo de internação, menos dor pós-operatória e até redução de $\operatorname{custos}^{10}$. Por questões operacionais, nossa experiência em apendicectomia laparoscópica não é grande. Realizamos poucos e selecionados casos que não foram incluídos nessa série.

O uso mais freqüente de incisão mediana no grupo > 40 anos demonstra uma maior dúvida diagnóstica, provavelmente em pacientes com tempo de evolução no mínimo semelhante, já que a ocorrência de supuração foi maior nos pacientes mais jovens. A incidência de apendicite aguda foi a mesma entre os sexos, mas o tempo de internação foi maior. Os nossos resultados complementam dados da literatura, alertando a ocorrência de apendicite aguda também em pacientes idosos.

\section{CONCLUSÃO}

A apendicite aguda em pacientes acima de 40 anos e especialmente acima dos 60 anos de idade demanda uma atenção especial. Os pacientes são em geral tratados em fase mais tardia, forçando o uso mais freqüente da laparotomia mediana. $\mathrm{O}$ aumento da morbidade, o maior tempo de internação e a elevação nos custos poderiam ser minimizados. Precisamos lembrar da hipótese de apendicite nos casos de abdômen agudo, principalmente nos pacientes mais idosos.

SUMMARY: Introduction: The low frequency of acute appendicitis in patients over 40 years old suggests that a better analysis is necessary. Method: We have made a Cohort in 1343 patients submitted to appendectomy from 2001 to 2005 . Patients were divided in two groups: over $(n=217)$ and under $(n=1126) 40$ years old. The Evangelico Hospital Colorectal Data Base was accessed to give surgical, histopathologic and clinical outcome. Results: In the over 40 group, surgical findings demonstrated edematous appendicitis in $\mathbf{4 2 . 8 5} \%$ and suppuration in $\mathbf{2 6 . 7 2 \%}$. Median incision was used in 39.17\% and the average hospital stay was 4.65 days. In the under 40 group, surgical findings demonstrated edematous appendicitis in $24.5 \%$ and suppuration in 32.6\%. Median incision was used in $11.90 \%$ and average admission time was 3.35 days. Conclusion: Patients over 40 presented more complexes cases, probably because of the low suspicion index.

Key words: appendicitis; appendectomy; aged; surgery

\section{REFERÊNCIAS BIBLIOGRÁFICAS}

1. Hardin, D., Acute appendicitis: review and update. American Family Physician, 1999. 60(7): p. 2027-34.

2. Storm-Dickerson TL, H.M., What have we learned over the past 20 years about appendicitis in the elderly? The American Journal of Surgery, 2003. 185: p. 198-201.
3. Hale D, M.M., Pearl R, Schutt D, Jaques D., Appendectomy: A contemporary Appraisal. 1997. 225(3): p. 252-261.

4. Blomqvist PG, A.R., Granath F, Lambe MP, Ekbom AR. ., (): Mortality After Appendectomy in Sweden. 233, 2001. 4: p. $455-460$.

5. Gupta H, D.D., Abdominal emergencies: has anything changed? Surg Clin North Am, 1997. 77: p. 1245-64. 
6. Giulinano V, G.C., Pinto F, Scaglione M, CT method for visualization of the appendix using a fixed oral dosage of diatrizoate-clinical expirience in 525 cases. Emerg Ragiol, 2005. 11(5): p. 281-5.

7. Weston AR, J.T., Blamey S, Diagnosis of appendicitis in adults by ultrasonography or computed tomography: a systematic review and meta-analysis. Int J Technol Assess Health Care, 2005. 21(3): p. 368-79.

8. Tzamakis NE, E.S., Danolidis K, Rallis GE, Tsioulos DI, Chatzivasiliou A, Peros G, Nikiteas NI, A new approach to accurate diagnosis of acute appendicitis. World J Surg, 2005. 29(9): p. 1151-6.

9. Yang HR, W.Y., Chung PK, Jeng LB, Chen RJ, Role of leukocyte count, neutrophil percentage, and C-reactive protein in the diagnosis of acute appendicitis in the elderly. Am Surg, 2005. 71(4): p. 344-7.
10. Sauerland S, L.R., Neugebauer EAM, Laparoscopic versus open surgery for suspected appendicitis (Cochrane Review). The Cochrane Library, 2004(2).

Endereço para correspondência: ANTONIO SÉRGIO BRENNER

Rua da Paz, 156

80.060-160 - Curitiba (PR)

fax: 4132629865

E-mail: asbrenner@ufpr.br 